\title{
BOUNDING CODIMENSION-ONE SUBVARIETIES AND A GENERAL INEQUALITY BETWEEN CHERN NUMBERS
}

\author{
By Steven Shin-Yi Lu and Yoichi MiYaoka
}

\begin{abstract}
We extend the Miyaoka-Yau inequality for a surface to an arbitrary nonuniruled normal complex projective variety, eliminating the hypothesis that the variety must be minimal. The inequality is sharp in dimension three and is also sharp among minimal varieties. For nonminimal varieties in dimension four or higher, an error term is picked up which can be controlled. As a consequence, we bound codimension one subvarieties in a variety of general type linearly in terms of their Chern numbers. In particular, we show that there are only a finite number of smooth Fano, Abelian and Calabi-Yau subvarieties of codimension one in any variety of general type.
\end{abstract}

1. Introduction. Investigation on the general behavior of subvarieties in an arbitrary algebraic variety has been a difficult but classical subject. The lack of very general techniques often restricts the investigation to very specific examples. Still, many patterns emerged which form the basis of some very strong conjectures. The aim of this paper is to address one of these conjectures quoted below by giving a very general Miyaoka-Yau type inequality valid for an arbitrary nonuniruled (logarithmic) variety in characteristic zero (see Theorem 3 and its corollary for the precise statements).

We begin with some definitions. We will work over an algebraically closed field of characteristic zero. Let $X$ be a projective variety. A vector sheaf, also known as a locally free sheaf, $\mathcal{E}$ on $X$ is called big if some symmetric power $S^{k} \mathcal{E}$ of $\mathcal{E}$ contains an ample subsheaf. This is, of course, equivalent to the usual definition via the bigness of the tautological line sheaf $\mathcal{O}_{E}(1)$, where $\mathcal{E}=\mathcal{O}(E) . X$ is said to be of general type if a nonsingular model of $X$ has big canonical line sheaf. From now on, we use the notation $K_{M}$ to denote a divisor corresponding to the canonical line sheaf of an algebraic manifold $M$ and call $-K_{M}$ the anticanonical divisor of $M$.

A basic motivation for our investigation is the following sweeping conjecture of Serge Lang [L1]:

Manuscript received May 15, 1995; revised April 17, 1996.

Research of the first author supported in part by a JSPS fellowship and JSTF.

American Journal of Mathematics 119 (1997), 487-502. 
Geometric Lang's Conjecture. Let $X$ be a variety of general type. Then a proper Zariski closed subset of $X$ contains all its subvarieties not of general type. In particular, $X$ has only a finite number of codimension-one subvarieties not of general type.

Except for a result of Bogomolov for a certain class of surfaces, very little is known so far in the general direction of this conjecture. It is noteworthy in passing that the validity of this conjecture has been used to establish a strong result in arithmetic geometry of the existence of an universal upper bound for the number of rational points for curves over number fields (see [CHM]). We will address the problem of effective upper bounds for the same problem, but for the complex function field case, in a separate paper.

We first quote the following theorem in dimension two (see [LM] for details).

THEOREM 1. In a surface $S$ of general type, there are only a finite number of rational curves and elliptic curves with a bound on the number of ordinary nodes of each curve.

In higher dimensions, the following result can be found in [Lu]. Please see the next section for the definition of a nef or pseudoeffective divisor.

THEOREM 2. If the cotangent sheaf of a nonsingular projective variety $X$ is big, then $X$ contains only a finite number of codimension-one subvarieties whose desingularizations do not have big cotangent sheaves. In particular, X has only a finite number of codimension-one subvarieties each with a desingularization having nef (more generally, pseudoeffective) anticanonical divisor.

This is just the forementioned theorem of Bogomolov in the surface case extended to arbitrary dimensions. For surfaces of general type, the condition $c_{1}^{2}>c_{2}$ guarantees the bigness of the cotangent sheaf, which is the hypothesis of the above theorem.

We stress that the hypothesis of this theorem holds for a large fraction of varieties of general type and, modulo the minimal model conjecture (MMP) for which we refer to $([\mathrm{KMM}])$, implies that the variety is of general type. For this reason, the conclusion of the theorem (modulo the MMP) includes those subvarieties not of general type, which yields Lang's conjecture in codimension one in this case.

In this paper, we deal with all varieties of general type but restrict our attention to nonsingular codimension-one subvarieties for simplicity. We first note the trivial fact (see Proposition 1) that a variety of general type cannot be uniruled, i.e., cannot have a rational curve through a generic point. Our main results here are as follows.

THEOREM 3. Let $X$ be a nonsingular projective variety of dimension $n \geq 2$. Let its canonical divisor be $K$. Let $H$ be a nef divisor. Let $D$ be a reduced irreducible 
divisor which, as a variety, is nonsingular and whose anticanonical divisor $-K_{D}$ is pseudoeffective. If $X$ is not uniruled, then

$$
\frac{1}{2} H^{n-2} K D \leq H^{n-2}\left\{3 c_{2}(X)-c_{1}^{2}(X)\right\}+C(H),
$$

where $C(H)$ is a constant depending only and linearly on $H^{n-2}, C(H)=0$ when $K$ is nef, and where $C(H)$ and the factor $\frac{1}{2}$ can be dropped in the case $n=2$ or 3 or when $K+D$ is nef.

We remark that, interestingly, the appearance of the factor $\frac{1}{2}$ and the constant $C(H)$ is in fact necessary. One way to see this is to compute the Chern numbers of the log-complex along the exceptional divisor of the blowing up of a point on an abelian variety of dimension four or higher.

COROLlary 1. Let $X$ be a nonsingular projective variety. If $X$ is of general type, then $X$ has only a finite number of nonsingular codimension-one subvarieties having pseudoeffective anticanonical divisor. In particular, $X$ has only a finite number of nonsingular codimension-one Fano, Abelian, and Calabi-Yau subvarieties.

The key to our result is a very general and powerful Miyaoka-Yau type inequality which, for simplicity, we only state in dimension three:

THEOREM 4. Let X be a nonsingular three dimensional projective manifold. Let $D$ be a reduced and irreducible divisor with only normal crossing singularities. Let $\Omega_{X}(\log D)$ be the logarithmic cotangent sheaf of $X$ along the divisor D. If $X$ is not uniruled, then

$$
c_{1}^{2}\left(\Omega_{X}(\log D)\right) H \leq 3 c_{2}\left(\Omega_{X}(\log D)\right) H
$$

for any nef divisor $H$ on $X$.

The bulk of the proof of Theorem 4 and its higher dimensional analogue, Theorem 3, follows that of Miyaoka in [M2]. We emphasize that the inequality in this theorem holds for arbitrary polarizations $H$ and is expected to have many applications besides the special focus of this paper. In particular, it is hoped that further insights into the Minimal Model Program of Mori may be gained through it as this theorem in the case of nef $K_{X}+D=\operatorname{det}\left(\Omega_{X}(\log D)\right)$ was a crucial ingredient in proving the validity of the Minimal Model Conjecture in dimension three. Indeed, a good estimate for $C(H)$ would have implications for the Mimimal Model Problem.

The analogue of Theorem 4 in dimension two can be found in [M1] and is the basic tool used in establishing a form of Theorem 3 for arbitrary divisors on surfaces in [LM]. It is worth mentioning that [M1] gave a lower bound for $3 c_{2}\left(\Omega_{X}(\log D)\right)-c_{1}^{2}\left(\Omega_{X}(\log D)\right)$ by the absolute value of the self-intersection of the negative part of the Zariski decomposition of $K_{X}+D$. In this respect, Theorem 
3 is stronger than Theorem 4 in that Theorem 3 gives a lower bound (namely $K_{X} D$ where $D$ is any smooth codimension-one subvariey with pseudoeffective anticanonical divisor) for $3 c_{2}(X)-c_{1}^{2}(X)$ in dimension three.

We should mention that for $(X, D)$ as in Theorem 4 with ample $K=K_{X}+D$, a stronger inequality

$$
3 c_{1}^{2}\left(\Omega_{X}(\log D)\right) K \leq 8 c_{2}\left(\Omega_{X}(\log D)\right) K
$$

holds by S.-T. Yau's fundamental work on the existence of Kahlër-Einstein metrics, with the equality case characterizing quotients of the unit ball in $\mathbf{C}^{3}$ (see [TY], [Ts1] and also [CY, Y3]). In fact, thanks to the validity of the Minimal Model Conjecture in dimension three, this stronger inequality holds for any threefold of general type if $K$ in the inequality is replaced by the pull back (implicitly up to a modification of $X$ ) of the canonical divisor of the minimal model (c.f. [LT] where the inequality is established directly on a minimal variety with equality characterizing ball-quotients). We note that the pull back (up to a modification) of the canonical divisor from the minimal model, if one exists, is just the positive part of the rational Zariski decomposition of the canonical divisor (pulled back via the modification) for any variety of general type. Incidentally, Tsuji [Ts2] has claimed an analytic form of Zariski decompositon over $\mathbf{R}$ using Kähler-Einstein metrics, which one can see directly, in fact, using the Yau volume form introduced in [Y0] according to him.

Acknowledgments. The first author would like to thank Professors S.-T. Yau and Barry Mazur for conversations concerning the results of this paper.

\section{Some preliminaries}

2.1. Pseudoeffectivity. Let $X$ be an $n$-dimensional nonsingular projective variety over an algebraically closed field $k$ of characteristic zero. We may set $k=\mathbf{C}$ without loss of generality. By a divisor on $X$, we will always mean a rational linear combination of codimension-one subvarieties of $X$ and it is called effective (repectively integral) if all coefficients are nonnegative (respectively integral). This notion is equivalent to that of $\mathbf{Q}$-divisors in the literature. Intersection of divisors and cycles are written multiplicatively. The reader should be cautioned that one often uses the same notation for a line sheaf and an integral divisor which gives rise to it, and that we adopt this usage here.

Definition 1. A divisor $D$ on $X$ is called numerically effective (respectively, pseudo-effective) if $D C \geq 0$ for all curves $C$ in $X$ (respectively, if $D H^{n-1} \geq 0$ for all ample divisors $H$ on $X$ ). Numerically effective divisors are called $n e f$ for short. 
It is instructive to compare the definition of being nef with Kleiman's criterion for ampleness ([K1]) which we do not state. An easy consequence of this criterion is that if $D$ is a pseudoeffective divisor, then $D P^{n-1} \geq 0$ for all nef divisors $P$. We should mention that our definition of a pseudo-effective divisor differs from the usual one where the $H^{n-1}$ above is generally allowed to be replaced by the intersection of $n-1$ distinct ample divisors.

We note that the notion of pseudoeffectivity is a numerical one and makes sense on the group $\operatorname{Pic}(X)$ of integral divisors modulo linear equivalence. Also, this notion extends naturally to the Chow ring of cycles of arbitrary codimension modulo rational equivalence and is often referred to as semipositivity. Incidentally, Chern classes of vector bundles over $X$ are regarded as elements of the Chow ring.

We first examine the pseudoeffectivity of the canonical divisor $K_{X}$ of $X$. The following is a corollary of an important theorem of Mori found in [MM]:

THEOREM 5 (Mori-Miyaoka). If there is a Zariski open set $\mathcal{U}$ in $X$ such that for every point $x$ in $\mathcal{U}$ there is a curve $C$ containing $x$ such that $K_{X} C<0$, then $X$ is uniruled.

Hence if $X$ is not uniruled, then $K_{X}$ is pseudoeffective. The converse of this statement is also true and follows from the general theory. We will be content here to work out only the following trivial partial converse.

Proposition 1. If $K_{X}$ is effective (meaning $\mathbf{Q}$-effective), then $X$ is not uniruled.

Proof. Assume $K_{X}$ is effective. If $X$ is uniruled, then there exist a generically finite and dominating map $\pi: V \rightarrow X$ where $V$ admits a fibration $p: V \rightarrow B$ with $\mathbf{P}^{1}$ as generic fibres. This is because the Hilbert scheme of rational curves has at most countably many components but $X$ is uncountable. Clearly $\pi^{*} K_{X}$ is effective so that $K_{V}$ must be also as $K_{V}-\pi^{*} K_{X}$ is effective. Hence $\left.K_{V}\right|_{F}$ is efffective for the generic fiber $F$. But for the generic fiber $F,\left.K_{V}\right|_{F}=K_{F}$ by the adjunction formula and $K_{F}=\mathcal{O}_{\mathbf{P}^{1}}(-2)$, which is not effective! This is a contradiction.

The preceding theorem admits a very powerful generalization which we will come to in the next section.

We end this section with the following celebrated theorem of Zariski [Zar] in dimension two (c.f. Fujita $[\mathrm{Fu}]$ ).

Theorem 6 (Zariski Decomposition Theorem for Surfaces). Let $X$ be an algebraic surface. Any pseudo-effective divisor $D$ on $X$ has a unique decomposition $D=P+N$ where $P$ and $N$ are divisors, $P$ is nef, and either $N=0$ or $N$ is effective and $P$-exceptional.

This decomposition is called the Zariski decomposition of $D . P$ is called the positive part of $D$ and $N$ the negative or the exceptional part. By Grauert's 
criterion, a divisor $N$ is exceptional if and only if the intersection matrix of the irreducible components of $N_{\text {red }}$ is negative definite. We call a divisor $P$ exceptional if it is exceptional and the intersection of any of its component with $P$ is zero. We will have occasion to use a basic lemma from Zariski's original proof of this theorem, for which we quote Corollary 7.2 of [Zar]:

Lemma 1. Let $Z=\sum a_{i} E_{i}$ be an exceptional divisor on a surface. If $D$ is an effective (respectively, pseudoeffective) divisor such that $(D-Z) E_{i} \leq 0$ for all $i$, then $D-Z$ is effective (respectively, pseudoeffective).

In higher dimensions, the analogue of this decomposition theorem for the canonical divisor of a nonuniruled variety is at the heart of the Minimal Model Program.

2.2. Semistability and generic semipositivity. The cotangent sheaf of a nonuniruled variety satisfies some very deep geometric properties which we will come to after some definitions. Let us first fix a nonsingular projective variety $X$ of dimension $n$. Fix also a nef divisor $P$ on $X$, which we call a polarization.

Definition 2. A torsion-free coherent sheaf $\mathcal{E}$ on $X$ is called semistable (respectively, seminegative) with respect to $P$ if whenever $\mathcal{G}$ is a nonzero coherent subsheaf of $\mathcal{E}$, we have

$$
\frac{c_{1}(\mathcal{G}) P^{n-1}}{\operatorname{rank} \mathcal{G}} \leq \frac{c_{1}(\mathcal{E}) P^{n-1}}{\operatorname{rank} \mathcal{E}}\left(\text { respectively, } c_{1}(\mathcal{G}) P^{n-1} \leq 0\right) .
$$

$\mathcal{E}$ is called semipositive with respect to $P$ if its dual $\mathcal{E}^{\vee}$ is seminegative with respect to $P$.

The definition extends naturally to the case where $P^{n-1}$ is replaced by the intersection of $n-1$ polarizations. This definition of semistability via degree is due to Mumford and Takemoto in the case of ample $P$ and we note its equivalence with seminegativity, or semipositivity when $c_{1}(\mathcal{E}) P^{n-1}=0$.

Definition 3. A torsion-free coherent sheaf on $X$ is called generically semipositive if it is semipositive with respect to an arbitrary polarization $P$ on $X$.

This definition differs slightly from the original in [M2], but is simpler and effectively the same. We note that $\mathcal{E}$ is generically semipositive if and only if all its torsion-free quotients have pseudoeffective determinant line sheaves. Hence, torsion free quotients of a generically semipositive sheaf are also generically semipositive.

The following generalizes Theorem 5 and is a crucial ingredient in the proof of our higher dimensional Chern-number inequality and our main theorem. See Miyaoka [M2] for the main part of the proof. 
Proposition 2 (Generic semipositivity of cotangent sheaves). Let $X$ be an $n$ dimensional projective manifold, and $D$ a reduced divisor with only normal crossing singularities. If $X$ is not uniruled, then the log-cotangent sheaf $\Omega_{X}(\log D)$ of $X$ along $D$ is generically semipositive.

Proof. The case when $D$ is empty has already been established in [M2]. For the general case, we consider the inclusion $0 \longrightarrow \Omega_{X} \longrightarrow \Omega_{X}(\log D)$. Let $Q$ be a torsion-free quotient of $\Omega_{X}(\log D)$. Then $Q$ natually contains a torsion free quotient $Q_{0}$ of $\Omega_{X}$ and $Q$ is isomorphic to $Q_{0}$ stalkwise outside $D$. Hence $\operatorname{det} Q_{0}$ natually injects to $\operatorname{det} Q$. But $\operatorname{det} Q_{0}$ is pseudoeffective by the generic semipositivity of $\Omega_{X}$ and thus so is $\operatorname{det} Q$. This establishes the proposition.

We will need a slight refinement of the above proposition. But we first recall the basic fact that given any torsion-free coherent sheaf $\mathcal{E}$ and a polarization $P$, there exists a unique maximal semistable subsheaf (also known as the maximal destabilizing subsheaf) with respect to $P$ and which is commonly denoted by $\mathcal{E}_{1}$ (being the first term in the filtration of Harder and Narasimhan [HN]). It is characterized by the property that

$$
\frac{c_{1}(\mathcal{G}) P^{n-1}}{\operatorname{rank} \mathcal{G}} \leq \frac{c_{1}\left(\mathcal{E}_{1}\right) P^{n-1}}{\operatorname{rank} \mathcal{E}_{1}}
$$

for every coherent subsheaf $\mathcal{G}$ of $\mathcal{E}$ with equality forcing $\mathcal{G}$ to be a subsheaf of $\mathcal{E}_{1}$. Note therefore that $\mathcal{E}$ is seminegative with respect to $P$ if and only if $c_{1}\left(\mathcal{E}_{1}\right) P^{n-1} \leq 0$. Now a deep theorem of Mumford and Mehta-Ramanathan [MR] claims that the maximal destabilizing subsheaf of the restriction of a torsion free coherent sheaf $\mathcal{E}$ on $X$ to a generic hypersurface section $Y$ of high degree must be the restriction of the maximal destabilizing subsheaf of $\mathcal{E}$ to $Y$. We underline this as follows:

Remark 1. $\left.\mathcal{E}_{1}\right|_{Y}=\left(\left.\mathcal{E}\right|_{Y}\right)_{1}$ for a generic hypersurface section $Y$ of high enough degree.

Proposition 3. Let $X$ and $D$ be as before, $\mathcal{E}=\Omega_{X}(\log D)$. Let $H$ be a very ample divisor. If $m$ is a sufficiently large integer and $S$ a sufficiently general surface section cut out by members of $|m H|$, then the restriction $\left.\mathcal{E}\right|_{S}$ of $\mathcal{E}$ to $S$ is generically semipositive. Furthermore, given a countable set $\mathcal{D}$ of such $D$ 's one can choose an $m$ and $a S$ so that for all $D \in \mathcal{D}$ we have that $\left.\mathcal{E}\right|_{S}=\left.\Omega_{X}(\log D)\right|_{S}$ is generically semipositive.

Proof of Proposition 3. We first deal with the case of one $D$. It is easy to see the pseudoeffectivity of the determinant $K_{X}+D$ of $\mathcal{E}$ when restricted to a general $S$ : If not, then $K_{X} C<0$ for a family of curves covering $S$ for general $S$ which would contradict the theorem of Miyaoka and Mori (Theorem 5) as $X$ 
is not uniruled. The general case is similar and requires the pseudoeffectivity of the determinant of other torsion free quotients of $\mathcal{E}$ when restricted to $S$.

Equivalently, we may consider the dual of $\mathcal{E}, \mathcal{E}^{\vee}=T_{X}(-\log D)$ : If $\left.\mathcal{E}^{\vee}\right|_{S}$ is not generically seminegative for general $S$, then neither is $\left.T_{X}\right|_{S}$. So for each such $S$ there is a very ample divisor $H_{S}$ on $S$ such that $\left(\left.T_{X}\right|_{S}\right)_{1}$ has positive degree when restricted to a generic member $C$ of $\left|m H_{S}\right|(m>0)$, which is a curve. But for $m \gg 0$, we have $\left.\left(\left.T_{X}\right|_{S}\right)_{1}\right|_{C}=\left(\left.T_{X}\right|_{C}\right)_{1}$ for such a curve $C$ by Remark 1 . Such curves cover an open set of $X$ and thus allow us to apply the argument of Miyaoka-Mori as in [M3] (even though the family may not be bounded apriori) to conclude that $X$ is uniruled, contrary to our assumption.

We now consider the case of a countable set $\mathcal{D}$ of normal crossing divisors. We have shown, by above, that there is an $m$ such that for a general 2-dimensional section $S$ cut out by members of $|m H|$ we have that $\left.\Omega_{X}\right|_{S}$ is generically semipositive. As $m H$ is very ample, the general surface section $S$ of $|m H|$ is not contained in any $D \in \mathcal{D}$. With such a choice, the following inclusion of sheaves

$$
\left.\left.0 \longrightarrow \Omega_{X}\right|_{S} \longrightarrow \Omega_{X}(\log D)\right|_{S}
$$

holds for all $D \in \mathcal{D}$. As the above inclusion is actually an isomorphism on the nonempty open set $S \backslash D$ for each $D$, the same argument as in Proposition 2 shows that $\left.\Omega_{X}(\log D)\right|_{S}$ is also generically semipositive.

We now quote an important theorem on semistability ([B2, Gi], see [M2] for an elementary proof), which we will need.

THEOREM 7 (The Bogomolov-Gieseker inequality). Let $\mathcal{E}$ be a torsion free coherent sheaf of rank $r$ on a projective surface with a nef divisor $P$. If $P$ is not numerically trivial and $\mathcal{E}$ is semistable with respect to $P$, then

$$
2 r c_{2}(\mathcal{E}) \geq(r-1) c_{1}^{2}(\mathcal{E})
$$

We note that if $\mathcal{E}$ is the (co)tangent sheaf of an $n$-dimensional projective manifold with ample canonical divisor $K$, then $\mathcal{E}$ is semistable with respect to $K$ thanks to Yau's resolution of the Calabi conjecture. Also, the stronger inequality (for rank $n$ )

$$
2(n+1) c_{2}(\mathcal{E}) K^{n-2} \geq n c_{1}^{2}(\mathcal{E}) K^{n-2},
$$

holds in this case ([Y1, Y2]). This stronger inequality can be established for any variety (up to a modification) provided it has a minimal model and where $K$ is replaced by a suitable pull back of the canonical divisor from the minimal model (see [LT]). 
3. Semipositivity of $3 c_{2}-c_{1}^{2}$ and an application. The following theorems are extracted from [M2].

THEOREM 8. Let $\mathcal{E}$ be a vector sheaf on a surface $S$. Let $K=\operatorname{det} \mathcal{E}$. Assume $K$ is nef. If $K$ is numerically equivalent to zero, let $P$ be any nef divisor not numerically equivalent to zero. Otherwise, let $P=K$. If $\mathcal{E}$ is semipositive with respect to $P$, then

$$
c_{2}(\mathcal{E}) \geq 0
$$

THEOREM 9. The hypothesis and setup are as above but assume further that $S$ is not uniruled. In the case $K^{2}>0$ and rank $\mathcal{E}_{1} \leq 2$, assume also that there is an injection $\mathcal{E}_{1} \rightarrow \Omega_{S}(\log D)$ for some normal crossing divisor $D$ on $S$. If $\mathcal{E}$ is semipositive with respect to $P$, then

$$
3 c_{2}(\mathcal{E})-c_{1}^{2}(\mathcal{E}) \geq 0
$$

The proof of these theorems follows the exact same argument as that of Theorem 6.1 and Proposition 7.1 of [M2] in the case $P^{2}>0$, so we refer interested readers there. In the case $P^{2}=0$, one can still follow the argument of Theorem 6.1 of [M2] to some extent, but we offer a more direct argument here.

Proof of the theorems in the case $P^{2}=0$. Let $P^{2}=0$. Then $P K=0$ and $c_{1}^{2}(\mathcal{E})=K^{2}=0$ by assumption. Semipositivity of $\mathcal{E}$ with respect to $P$ implies that $c_{1}\left(\left(\mathcal{E}^{\vee}\right)_{1}\right) P \leq 0$. Now $c_{1}\left(\mathcal{E}^{\vee}\right) P=-K P=0$, so the properties of the maximal destabilizing subsheaf forces $\mathcal{E}^{\vee}=\left(\mathcal{E}^{\vee}\right)_{1}$. As the latter is semistable, the Bogomolov-Giesecker inequality applies to give

$$
c_{2}(\mathcal{E})=c_{2}\left(\mathcal{E}^{\vee}\right) \geq 0
$$

as desired.

Theorems 8 and 9 easily extend to the case where $\mathcal{E}$ is a $\mathbf{Q}$-vector sheaf. We recall that a $\mathbf{Q}$-vector sheaf has the form $\mathcal{F}(D)$ for a vector sheaf $\mathcal{F}$ and a (not necessarily integral) divisor $D$. The basic step in reducing a $\mathbf{Q}$-vector sheaf problem to a vector sheaf problem is the application of a standard but very useful covering trick:

LemMa 2 (Covering Lemma, c.f. [KMM, Theorem 1-1-1]). Let $X$ be a smooth projective variety. Let $D$ be a divisor such that $D_{\text {red }}$ has only (simple) normal crossing singularities. Then there is a finite Galois morphism $\pi: Y \rightarrow X$ from a nonsingular variety $Y$ such that $\pi^{*} D$ becomes an integral divisor.

If $\mathcal{F}$ is a vector sheaf and $D$ a divisor on a surface $X$, we may resolve the singularity of $D$ by a morphism $r: \tilde{X} \rightarrow X$ where $r$ is a composition of blowups 
and where $\tilde{X}$ is smooth. Then by applying the above lemma to $r^{*} D$, we get a Galois morphism $\pi: \tilde{Y} \rightarrow \tilde{X}$ with Galois group $G$ such that the pull back $\tilde{D}$ of $D$ under $\tau=\pi \circ r$ is integral. Consider a nef divisor $P$ on $X$ giving rise to a nef divisor on $\tilde{X}$ and on $\tilde{Y}$ by pulling-back. Now observe that $\mathcal{F}_{1}$, being reflexive, is locally free on $X$. Let $\tilde{\mathcal{F}}=r^{*} \mathcal{F}$. Then $r^{*}\left(\mathcal{F}_{1}\right)=\left(r^{*} \mathcal{F}\right)_{1}=(\tilde{\mathcal{F}})_{1}$ as $r$ is connected. Also $\pi^{*}\left(\tilde{\mathcal{F}}_{1}\right)=\left(\pi^{*} \tilde{\mathcal{F}}\right)_{1}$ because

$$
g^{*}\left(\pi^{*} \tilde{\mathcal{F}}\right)_{1}=\left(\pi^{*} \tilde{\mathcal{F}}\right)_{1}
$$

for all $g \in G$ by the uniqueness of $\left(\pi^{*} \tilde{\mathcal{F}}\right)_{1}$ in $\pi^{*} \tilde{\mathcal{F}}$. Hence, for $\mathcal{E}=\mathcal{F}(D)$, we see that $\tau^{*} \mathcal{E}=\left(\tau^{*} \mathcal{F}\right)(\tilde{D})$ is a vector sheaf on $\tilde{Y}$ and that

$$
\left(\tau^{*} \mathcal{E}\right)_{1}=\left(\tau^{*}\left(\mathcal{F}_{1}\right)\right)(\tilde{D})=\left(\tau^{*} \mathcal{F}\right)_{1}(\tilde{D})
$$

which therefore serves as a good substitute for " $\mathcal{E}_{1}$." So it is fitting to define $\mathcal{E}_{1}$ as the $\mathbf{Q}$-vector sheaf $\mathcal{F}_{1}(D)$, which is easily seen to be independent of the choice of $\mathcal{F}$ and $D$ to represent $\mathcal{E}$. We may now define the $\mathbf{Q}$-vector sheaf $\mathcal{E}$ to be semistable with respect to $P$ if $\mathcal{E}_{1}=\mathcal{E}$ and to be semipositive with respect to $P$ if $c_{1}\left(\left(\mathcal{E}^{\vee}\right)_{1}\right) P \leq 0$. We are now ready to give Theorems 8 and 9 for the case of $\mathbf{Q}$-vector sheaves. Only a proof in the case $P^{2}>0$ will be offered as it is the only case we will use.

THeOrem 8 . Let $\mathcal{E}$ be a $\mathbf{Q}$-vector sheaf on a surface $S$. Let $K=\operatorname{det} \mathcal{E}$. Assume $K$ is nef. If $K$ is numerically equivalent to zero, let $P$ be any nef divisor not numerically equivalent to zero. Otherwise, let $P=K$. If $\mathcal{E}$ is semipositive with respect to $P$, then

$$
c_{2}(\mathcal{E}) \geq 0
$$

Proof in the case $K^{2}>0\left(P^{2}>0\right)$. Let $\tilde{\mathcal{E}}=\tau^{*} \mathcal{E}$ be the locally free sheaf on $\tilde{Y}$ given by the construction above, where we identified $X$ with $S$. Note that $\operatorname{det} \tilde{\mathcal{E}}=\tau^{*} K=\tilde{K}$ is nef and that $\tilde{\mathcal{E}}$ is semipositive with respect to $\tilde{K}$. Let

$$
0=\tilde{\mathcal{E}_{0}} \subset \tilde{\mathcal{E}_{1}} \subset \cdots \subset \tilde{\mathcal{E}_{s}}=\tilde{\mathcal{E}}
$$

be the semistable filtration of $\tilde{\mathcal{E}}$ with respect to $\tilde{K}$. Put $\mathcal{G}_{i}=\tilde{\mathcal{E}_{i}} / \tilde{\mathcal{E}_{i-1}}, r_{i}=\operatorname{rank} \mathcal{G}_{i}$, $r=\operatorname{rank} \tilde{\mathcal{E}}$. If $d$ is the generic degree of $\tau$, then $c_{2}(\tilde{\mathcal{E}})=d c_{2}(\mathcal{E})$ and $\tilde{K}^{2}=c_{1}^{2}(\tilde{\mathcal{E}})=$ $d c_{1}^{2}(\mathcal{E})$. In particular, $\tilde{K}$ is not numerically trivial and the Bogomolov-Gieseker inequality gives

$$
2 c h_{2}\left(\mathcal{G}_{i}\right)=2 c_{2}\left(\mathcal{G}_{i}\right)-c_{1}^{2}\left(\mathcal{G}_{i}\right) \geq-\frac{1}{r_{i}} c_{1}^{2}\left(\mathcal{G}_{i}\right)
$$


As $c h_{2}$ is additive with respect to direct sums of vector bundles, we get

$$
2 c_{2}(\tilde{\mathcal{E}})-c_{1}^{2}(\tilde{\mathcal{E}})=\sum_{i} 2 c h_{2}\left(\mathcal{G}_{i}\right) \geq-\sum_{i} \frac{1}{r_{i}} c_{1}^{2}\left(\mathcal{G}_{i}\right)
$$

Define $\alpha_{i}$ via $\alpha_{i} \tilde{K}^{2}=\frac{1}{r_{i}} c_{1}\left(\mathcal{G}_{i}\right) \tilde{K}$. Then we have $\alpha_{1}>\cdots \alpha_{s} \geq 0$, where the last inequality follows from the semipositivity of $\tilde{\mathcal{E}}$ with respect to $\tilde{K}$. Note also that $\sum_{i} r_{i} \alpha_{i}=1$ and that $\alpha_{1} \leq 1$ as a result. The Hodge index theorem gives

$$
c_{1}^{2}\left(\mathcal{G}_{i}\right) \tilde{K}^{2} \leq\left[c_{1}\left(\mathcal{G}_{i}\right) \tilde{K}\right]^{2}=r_{i}^{2} \alpha_{i}^{2}\left(\tilde{K}^{2}\right)^{2} .
$$

Hence, we obtain

$$
\begin{aligned}
2 c_{2}(\tilde{\mathcal{E}}) & \geq c_{1}^{2}(\tilde{\mathcal{E}})-\sum_{i} \frac{1}{r_{i}} c_{1}^{2}\left(\mathcal{G}_{i}\right) \\
& \geq\left(1-\sum_{i} r_{i} \alpha_{i}^{2}\right) \tilde{K}^{2} \\
& \geq\left\{1-\left(\sum_{i} r_{i} \alpha_{i}\right) \alpha_{1}\right\} \tilde{K}^{2} \\
& =\left(1-\alpha_{1}\right) \tilde{K}^{2} \geq 0 .
\end{aligned}
$$

So $c_{2}(\mathcal{E}) \geq 0$ as desired.

Observe that we have followed exactly the proof of Theorem 6.1 of [M2] except that we have replaced $\mathcal{E}$ by $\tilde{\mathcal{E}}$ in the above proof.

THEOREM 9'. The hypothesis and setup are as above but assume further that $S$ is not uniruled. In the case $K^{2}>0$ and rank $\mathcal{E}_{1} \leq 2$, assume also that there is an injection (as $\mathbf{Q}$-sheaves) $\mathcal{E}_{1} \rightarrow \Omega_{S}(\log D)$ for some normal crossing divisor $D$ on $S$. If $\mathcal{E}$ is semipositive with respect to $P$, then

$$
3 c_{2}(\mathcal{E})-c_{1}^{2}(\mathcal{E}) \geq 0
$$

The proof of Theorem $9^{\prime}$ is again exactly the same as the proof of Proposition 7.1 of [M2], except that we pull back $\mathbf{Q}$-vector sheaves to $\tilde{Y}$ via $\tau$ where they become vector sheaves and work on $\tilde{Y}$ as in the last proof.

We are now ready to prove our main theorems.

\subsection{Proof of the inequalities.}

Proof of Theorem 4. Let $\bar{\Omega}=\Omega_{X}(\log D)$. Let $\Delta$ be the Chern class $3 c_{2}-c_{1}^{2}$. We only need to prove the inequality $\Delta(\bar{\Omega}) H \geq 0$ for an arbitrary ample divisor 
$H$. This is because the required result is only numerical, i.e., it involves only intersection numbers, and Kleiman's criterion allows us to approximate any nef divisor by ample divisors that are arbitrarily close to it.

Given $H$, let $S$ be a generic member of $|m H|$ for some $m$ so that $m H$ is very ample. We need to show the nonnegativity of $\Delta\left(\left.\bar{\Omega}\right|_{S}\right)=m \Delta(\bar{\Omega}) H$ :

By Proposition 3 and Bertini's theorem, we may choose $m$ large enough and $S$ general enough so that $S \cap D$ is a smooth and irreducible divisor in $D$ and that $\left.\bar{\Omega}\right|_{S}$ is generically semipositive. By Remark 1, we may further choose $S$ general enough so that $\left(\left.\bar{\Omega}\right|_{S}\right)_{1}$ (in the case its rank is 1 or 2) injects to $\Omega_{S}\left(\left.\log D\right|_{S}\right)$ (by choosing $S$ to be transversal at some point to the foliation defined by $\bar{\Omega}_{1}$ on $X$ ). In particular, det $\left.\bar{\Omega}\right|_{S}$ is pseudoeffective and thus has a Zariski decomposition $P+N$.

Let $\mathcal{E}$ be the $\mathbf{Q}$-vector sheaf $\left.\bar{\Omega}\right|_{S}(-N / 3)$. We note that $\operatorname{det} \mathcal{E}=\left.\operatorname{det} \bar{\Omega}\right|_{S}-N=P$ as $\bar{\Omega}$ has rank three. Note also that $\mathcal{E}$ is semipositive with respect to $P$ by the generic semipositivity of $\left.\bar{\Omega}\right|_{S}$ and as $P N=0$. If $P$ is numerically trivial, we simply replace $\bar{\Omega}$ by $\bar{\Omega}\left(\epsilon H^{\prime}\right)$ for some small rational number $\epsilon$ (which we let approach zero) and some ample divisor $H^{\prime}$ on $X$. Note that $\bar{\Omega}\left(\epsilon H^{\prime}\right)$ is generically semipositive. This will guarantee that $P$ is not numerically trivial. With this setup, the hypotheses of the above theorems are satisfied so that we may conclude $\Delta(\mathcal{E}) \geq 0$. Now $\Delta$ is invariant under tensoring $\mathcal{E}$ by line sheaves as $\mathcal{E}$ has rank 3. Hence $\Delta\left(\left.\bar{\Omega}\right|_{S}\right) \geq 0$ as required.

Proof of Theorem 3. As before, we only need to prove it for the case where $H$ is ample.

The exact same argument as above works for a general surface $S$ cut out by the intersection of $n-2$ members of $|m H|$ for $m$ large enough for us to apply Theorems $8^{\prime}$ and $9^{\prime}$ and to conclude that $\Delta(\mathcal{E}) \geq 0$ where $\mathcal{E}=\left.\Omega_{X}(\log D)\right|_{S}(-N / n)$. As $\mathcal{E}$ has rank $n, \Delta$ is no longer invariant under tensoring by line sheaves for $n \neq 3$ but $3 c_{2}-\frac{3(n-1)}{2 n} c_{1}^{2}$ is. Let $\Delta_{0}=\Delta(\mathcal{E})$ and $\Delta_{1}=\Delta\left(\left.\Omega_{X}(\log D)\right|_{S}\right)$. An easy computation shows that $\Delta_{1}-\Delta_{0}=\frac{n-3}{2 n} N^{2}$. Hence,

$$
\Delta_{1}=3 c_{2}\left(\Omega_{X}(\log D)\right) S-\left(K_{X}+D\right)^{2} S \geq \frac{n-3}{2 n} N^{2} .
$$

We observe therefore that $\Delta_{1} \geq 0$ holds for $n \leq 3$ as $N^{2} \leq 0$, and also holds for $N=0$ (which is the case when $K_{X}+D=\operatorname{det} \bar{\Omega}$ is nef).

In order to bound $N^{2}$ from below, we assume for simplicity first that $K_{X}=$ $P^{\prime}+E$ where $P^{\prime}$ and $E$ are nef and effective divisors on $X$ respectively. This is certainly possible for the case we have in mind of general type $X$ and we remark that the bigger $P^{\prime}$ is with respect to the decomposition, the better is our bound for $C(H)$. Let $N=\sum_{i} a_{i} N_{i}$ and $E=\sum_{j} b_{j} E_{j}$ where $a_{i}$ and $b_{j}$ are positive rational numbers and $N_{i}, E_{j}$ are the irreducible components of $N_{\text {red }}$ and $E_{\text {red }}$ respectively. We may arrange $S$ so that it is not contained in $E_{\text {red }}$ in order that $\left.(E+D)\right|_{S}$ is effective. Since $P N_{i}=0$ for all $i$, Lemma 1 of Zariski applies to 
$\left.(E+D)\right|_{S}-N=P-\left.P^{\prime}\right|_{S}$ (by dotting it with $N_{i}$ ) forcing it to be effective (this can be observed directly from the definition of $P$ without Zariski's lemma of course). So we may write $N+F=\left.E\right|_{S}+\left.D\right|_{S}$ where all four terms in the equation are effective divisors. As a consequence, we see that

$$
N=\left.\left(c D+\sum_{j} c_{j} E_{j}\right)\right|_{S}
$$

where $0 \leq c \leq 1$ and $0 \leq c_{j} \leq b_{j}$ for all $j$. Note that either $\left.\left.D\right|_{S} \cdot E_{j}\right|_{S} \geq 0$ for all $j$ or $\left.D\right|_{S}=\left.E_{j}\right|_{S}$ for some $j$. Hence, squaring both sides of (+), we observe that

$$
N^{2}-c^{2} D^{2} S \geq-m^{n-2} C^{\prime}(H):=\min \left\{\sum_{j}\left(e_{j} E_{j} \mid S\right)^{2} \mid 0 \leq e_{j} \leq 1+c_{j}\right\} .
$$

If $K_{X} D S<0$, then we are done. Else, we have for $n>3$ that

$$
\begin{aligned}
\frac{1}{2} K_{X} D S+\frac{n-3}{2 n} N^{2} & \geq \frac{n-3}{2 n}\left(c^{2} K_{X} D S+N^{2}\right) \\
& \geq \frac{n-3}{2 n}\left[c^{2}\left(K_{X}+D\right) D S-m^{n-2} C^{\prime}(H)\right] .
\end{aligned}
$$

In the general case, the same argument works to arrive here except that the global decomposition $K_{X}=P^{\prime}+E$ must be replaced by the Zariski decomposition of $K_{X}$ when restricted to a suitable general surface section $S$ (for such a decomposition is unkown in higher dimensions for pseudoeffective canonical divisors). Now we must choose $S$ sufficiently generally to work independently of $D$. Note that the space of divisors on $X$ has at most countably many components. As we are only interested in numerical results, we only need to take a representative divisor from each component. Now $S$ can be chosen so that $S \cap D$ is smooth and irreducible for all chosen $D$ by Bertini's theorem and Baire's theorem. Also, by choosing $m$ large enough, $S$ can be chosen to be transversal at some point to the associated foliation defined by $\Omega(\log D)_{1}$ (in the case its rank is 1 or 2 ) and still be general enough so that $\left.\Omega(\log D)\right|_{S}$ is generically semipositive for all chosen $D$ by Proposition 3. Hence the same argument as in the last paragraph works to obtain $(*)$.

We have therefore established with $C(H)=\frac{n-3}{2 n} C^{\prime}(H)$ that for $n>3$,

$$
\frac{1}{2} K_{X} D S+\Delta_{1} \geq \frac{n-3}{2 n} c^{2}\left(K_{X}+D\right) D S-m^{n-2} C(H) .
$$

The rest of the theorem now follows by an easy computation using the pseudonegativity of $\left.\left(K_{X}+D\right)\right|_{D}=K_{D}$, namely, $\left(K_{X}+D\right) D S=m^{n-2} K_{D}\left(\left.H\right|_{D}\right)^{n-2} \leq 0$ : For 
$c_{1}\left(\Omega_{X}(\log D)\right)=K_{X}+D$ and $c_{2}\left(\Omega_{X}(\log D)\right)=c_{2}\left(\Omega_{X}\right)+\left(K_{X}+D\right) D$, so

$$
\Delta_{1}=\left[\Delta(X)+2\left(K_{X}+D\right) D-K_{X} D\right] S .
$$

This gives the theorem immediately in the case $n \leq 3$ and also in the case of nef $K_{X}+D$ as $\Delta_{1} \geq 0$ then. In the case $n>3,(* *)$ gives

$$
\frac{1}{2} K_{X} D S \leq\left[\Delta(X)+\left(2-\frac{n-3}{2 n} c^{2}\right)\left(K_{X}+D\right) D\right] S+m^{n-2} C(H) .
$$

So the desired inequality follows as $2-\frac{n-3}{2 n} c^{2}>1$. Note that when $K_{X}$ is nef, we can choose $E=0$ and therefore $C(H)=C^{\prime}(H)=0$ from the definition of $C^{\prime}(H)$.

\subsection{Boundedness and finiteness of $D$.}

Proof of Corollary 1. Let $H$ be an ample integral divisor. Let $l$ be such that $l K-H$ is linearly equivalent to an effective divisor $E$, which exists by the bigness of $K_{X}$. To bound the collection of divisors $D$ as given in the theorem, it is only necessary to bound the pair $\left(D,\left.H\right|_{D}\right)$ of $D$ as polarized varieties. The theorem of Matsusaka and Kollar ([KM]) says that it is enough to bound $\left(\left.H\right|_{D}\right)^{n-1}=H^{n-1} D$ and $\left(\left.H\right|_{D}\right)^{n-2} K_{D}=H^{n-2}\left(K_{X}+D\right) D$ for this purpose. By Theorem 3 , the former is bounded by 0 and $2 l\left[H^{n-2} \Delta(X)+C(H)\right]$ as long as $D$ is not a component of $E$. The latter is bounded by 0 and $-\Delta(X) H^{n-2}-C(H)$ (see the last inequality before this subsection) with the same proviso. The collection is therefore a bounded algebraic set. But this bounded algebraic set must be discrete as the easy addition law of Kodaira dimension for fibrations shows that $X$ is not of general type otherwise. Hence the collection is finite.

4. Conclusion. Although the full conjecture of Serge Lang is not known or reachable by present techniques, we hope that our modest result gives a good indication and pointer for the general conjecture and its future.

To conclude we will mention a few extensions and generalizations of our result, some other applications in the works, and some prospects for the future.

First of all, the Chern-number inequality obtained can be used to provide, for any divisor $D$, effective upper bounds for the degree of $D$ given in terms of the singularities of $D$ and lower bound for the degree of $D^{2}$ independent of the singularities as in the two dimensional case ([LM]). Secondly, we did not use the pseudoeffectivity of the anticanonical sheaf of $D$ in our proof of Theorem 3 except in the very final computation and it is clear that the theorem holds for general $D$ if we include some numerical or biregular invariant of some desingularization of $D$ and the contribution from the singularities, or if we have an upper bound on $D^{2} K_{X}^{n-2}$. Lastly, our results can easily be written in the logarithmic case to bound divisors in quasiprojective manifolds of log-general type. 
For applications, finding effective bounds for the number and height (canonical degree) of rational points over the function field of a curve of genus $g \geq 2$ has already been mentioned. The discreteness of certain codimension-two objects can be shown in certain special situations which is of interest. And effective bounds on the number of exceptional divisors for a variety of general type can be given via this approach.

As for the future, be it via analysis or algebra, special or general Chernnumber inequalities will always be useful in studying the geometry of algebraic varieties.

Pure Mathematics Department, University of Waterloo, Waterloo, Ontario, N2L 3G1 CANADA

RIMS, KYOTO UNIVERSITY, KYOTO, 606 JAPAN

REFERENCES

[B1] F. A. Bogomolov, Families of curves on a surface of general type, Soviet Math. Dokl. 18 (1977), 1294-1297.

[B2] Holomorphic tensors and vector bundles on projective varieties, Math. USSR-Izv. 13 (1978), 499-555.

[CHM] L. Caporaso, J. Harris, and B. Mazur, Uniformity of rational points, J. Amer. Math. Soc. 10 (1997), $1-35$.

[CY] S. Y. Cheng and S-T. Yau, Inequality between Chern numbers of singular Kähler surfaces and characterization of orbit space of discrete group of $\operatorname{SU}(2,1)$, Contemp. Math. 49 (1986), $31-43$.

[Fu] T. Fujita, On Zariski problem, Proc. Japan Acad. Ser. A Math. Sci. 55 (1979), 106-110.

[Gi] D. Gieseker, On a theorem of Bogomolov on Chern classes of stable bundles, Amer. J. Math. 101 (1979), 77-85.

[HN] G. Harder and M. S. Narasimhan, On the cohomology groups of moduli space of vector bundles on curves, Math. Ann. 212 (1975), 215-248.

[KMM] Y. Kawamata, K. Matsuda, and K. Matsuki, Introduction to the minimal model problem, Adv. Stud. Pure Math. 10 (1987), 283-360.

[K1] S. L. Kleiman, Toward a numerical theory of ampleness, Ann. of Math. 84 (1966), 293-344.

[KM] J. Kollar and T. Matsusaka, Riemann Roch type inequalities, Amer. J. Math. 105 (1983), 229-252.

[L1] S. Lang, Higher dimensional Diophantine problems, Bull. Amer. Math. Soc. 14 (1986), 159-205.

$[\mathrm{Lu}] \quad$ S. Lu, On meromorphic maps into varieties of log-general type, Proc. Sympos. Pure Math., vol. 52, Amer. Math. Soc., Providence, RI, 1991, 305-333.

[LM] S. Lu and Y. Miyaoka, Bounding curves in algebraic surfaces by genus and Chern numbers, Math. Res. Lett. 2 (1995), 663-676.

[LT] S. Lu and G. Tian, A general inequality of Chern numbers for minimal algebraic varieties and uniformization, CIMS preprint.

[MR] V. B. Mehta and A. Ramanathan, Semistable sheaves on projective varieties and their restriction to curves, Math. Ann. 258 (1982), 213-224.

[M1] Y. Miyaoka, The maximal number of quotient singularities on surfaces with given numerical invariants, Math. Ann. 268 (1984), 159-171. 
The Chern class and Kodaira dimension of a minimal variety, Adv. Stud. Pure Math. 10 (1987), 449-476.

[M3] Deformation of a morphism along a foliation and applications, Proc. Sympos. Pure Math., vol. 46, Amer. Math. Soc., Providence, RI, 1987, 245-268.

[MM] Y. Miyaoka and S. Mori, A numerical criterion for uniruledness, Ann. of Math. 123 (1986), 65-69.

[TY] G. Tian and S.-T. Yau, Existence of Kähler-Einstein metrics on complete Kähler manifolds and their applications to algebraic geometry, Mathematical Aspects of String Theory, World Scientific, River Edge, NJ, 1988, 574-672.

[Ts1] H. Tsuji, An inequality of Chern numbers for open algebraic varieties, Math. Ann. 277 (1987), 483-487.

[Ts2] _ Analytic Zariski decomposition, Proc. Japan Acad. Ser. A Math. Sci. 68 (1992), 161163.

[Y0] S.-T. Yau, Intrinsic measures on compact complex manifold, Math. Ann. 212 (1975), 317-329.

[Y1] Calabi's conjecture and some new results in algebraic geometry, Proc. Nat. Acad. Sci. U.S.A. 74 (1977), 1798-1800

[Y2] On the Ricci curvature of a compact Kähler manifold and the complex Monge-Ampère equation I, Comm. Pure Appl. Math. 31 (1978), 339-411.

[Y3] Métriques de Kähler-Einstein sur les variétés ouvertes, Astérisque 58 (1978), 163-167.

[Zar] O. Zariski, The theorem of Riemann-Roch for high multiples of an effective divisor on an algebraic surface, Ann. of Math. 76 (1962), 560-615. 